\title{
APUNTES PARA PENSAR EN UNA EDUCACIÓN NO SEXISTA
}

\author{
María Elena Acuña Moenne
}




\section{MARÍA ELENA ACUÑA MOENNE}

Antropóloga Social de la Universidad de Chile y doctora en Estudios Americanos, mención Pensamiento y Cultura, de la Universidad de Santiago de Chile. Actualmente es Profesora Asistente del Departamento de Antropología de la Universidad de Chile y se ha especializado en estudios de género, investigando sobre la reproducción de desigualdades de género y étnicas en la educación escolar. Participó en el año 2000 en la primera investigación sobre discriminación y segregación de género en la Universidad de Chile realizada por el Centro Interdisciplinario de Estudios de Género y en el año 2012 participó en la creación de la Oficina de Igualdad de Oportunidades de Género de la Universidad de Chile, que posteriormente dio origen a la actual Dirección de Igualdad de Género. 


\section{APUNTES PARA PENSAR EN UNA EDUCACIÓN NO SEXISTA}

\section{PRESENTACIÓN}

Durante el año 2018, en un contexto marcado por la emergencia de un movimiento feminista universitario con una fuerte expresión en la Universidad de Chile, se articula una demanda clara y contundente que plantea la necesidad de avanzar en una educación no sexista en la educación superior.

Los petitorios de los(as) estudiantes durante este movimiento identificaron como un obstáculo en la calidad de su formación y a la convivencia la existencia de un tipo de formación altamente "sexista", es decir, que discrimina, segrega y excluye a las mujeres y grupos LGTBI de diversas maneras, a través de las prácticas de enseñanza-aprendizaje que se modelan a través de diferentes sesgos, conductas que por lo demás, resultan muy difíciles de enumerar y, a veces, de identificar.

En este artículo intentaré establecer algunas líneas de reflexión sobre la posibilidad de avanzar en la construcción de una educación no sexista, primero considerando la historia general de la relación entre las mujeres y la formación universitaria, y luego esbozaré un conjunto de prácticas teóricas y además tomaré como ejemplo el proceso de análisis de la teoría antropológica realizado hace más de tres décadas por antropólogas feministas europeas y norteamericanas que deconstruyeron el canon ${ }^{1}$ teórico de esta disciplina, hasta ese entonces monolítico.

El propósito de esta reflexión es proponer que el movimiento académico político de construcción e instalación de una educación no sexista en primer lugar requiere de un marco mayor que apunte a establecer los múltiples modos en que las mujeres han sido excluidas y discriminadas en las universidades y que dentro de estas discriminaciones hay niveles epistémicos relacionados con la formación de las estructuras y corpus teóricos, lo que Celia Amorós (1985) llamara "la razón patriarcal”, así como también se expresan en los modos en que estructura un orden de género que condiciona el habitar diario de todos(as) los(as) miembros(as) de la comunidad.

En nuestro país hay un interés creciente en el debate sobre cómo los regímenes de género se expresan y afectan la presencia de mujeres y hombres en la educación

1. Por canon entendemos el conjunto de autores, teorías y temáticas que se reproducen cuando se apela a la historia de una disciplina. 
superior, en especial en las universidades: patrones de carreras académicas diferenciados entre las trayectorias masculinas y femeninas; acceso a cargos de toma de decisiones y liderazgos, presencia diferenciada de hombres y mujeres en áreas del conocimiento, malos tratos, abusos de poder, acoso sexual, discriminación de estudiantes LGTBI, exclusión del estatus de conocimiento -válido, por supuesto- a los estudios de género y a las teorías feministas, entre otras prácticas y expresiones que dan cuenta de la realidad de las relaciones de género en la educación superior. Con esto quiero decir que la preocupación por una educación no sexista debe ampliar su foco de discusión más allá del nivel del aula o del que estrictamente atañe a los procesos de enseñanza-aprendizaje.

Las discusiones al respecto se han orientado a realzar un momento inicial, fundacional, del ingreso femenino a las aulas universitarias a través de los relatos sobre las pioneras, las primeras en matricularse, en graduarse y tal vez, en ejercer una profesión. Estos estudios y reflexiones abordan el problema del olvido y las dificultades que las mujeres experimentaron en una etapa histórica de incorporación a las universidades, especialmente como académicas e investigadoras, generando una ruptura no sólo en términos de su presencia sino también en términos de los efectos que tiene para las mujeres el acceso a los espacios de producción y legitimación de los conocimientos socialmente relevantes. No obstante, también sabemos que la sola llegada de las mujeres a las universidades durante el siglo XX no cambió su estatus en la esfera de la producción de conocimientos científicos; es decir, a la par de la presencia material y corporal todavía quedaba en el horizonte una larga marcha para la transformación de las esferas simbólicas que reconociera la participación de las mujeres en la producción científica y en general, en la vida universitaria (Cordero, 2014; Cubillos y Monreal, 2013; Flecha, 2010; García, 2010; Magallón, 2010; Wolff, Winkler y Alvear, 2010; entre otros).

Un primer nivel de la presente reflexión se centrará en mostrar algunos elementos para comprender el orden de género de las universidades occidentalizadas (Grosfoguel, 2013); en un segundo nivel abordaré de manera más específica los códigos de género de la universidad y cómo estos van articulando lo que llamamos "educación sexista".

\section{ORDEN DE GÉNERO EN LA UNIVERSIDAD OCCIDENTALIZADA}

La presencia de hombres y mujeres en la universidad no es un fenómeno social estático sino más bien uno muy cambiante, y aunque suene una muletilla, es necesario insistir de manera permanente en su carácter histórico y situado, por lo que debe ser revisada constantemente para emprender el trabajo de urdir preguntas que nos 
aproximen a una reflexión que considere las actuales condiciones y características tanto del acceso de las mujeres a la educación superior como de su participación en las tareas y responsabilidades propiamente universitarias, así como las condiciones en que las mujeres realizan sus actividades en tanto estudiantes, académicas, investigadoras y profesionales en las universidades.

La ausencia y la presencia precaria de las mujeres en las universidades es, de acuerdo a Grosfoguel (2013), evidencia del racismo y sexismo epistémico fundacional de las estructuras de conocimiento de las universidades occidentalizadas; cómo se construye y funda el privilegio epistémico de los hombres blancos. Las preguntas fundamentales para poder entender cómo y por qué las universidades, en tanto instituciones sociales, han discriminado a las mujeres están relacionadas con la necesidad de comprender cómo los hombres monopolizaron tal autoridad y por qué lo que hoy conocemos como teoría social, histórica, filosófica o crítica se basa en la experiencia socio-histórica y la visión del mundo de hombres europeos y posteriormente norteamericanos provenientes de las elites. Es entonces la forma en la que se ha construido el conocimiento, los temas sobre los que se ha reflexionado y la construcción cultural de la fórmula del conocer hombre-razón, mujer-emoción, lo que está en el centro de la articulación de las universidades como instituciones generizadas, cuyas expresiones más concretas negocian y reflejan los modos particulares en que en cada momento histórico y en cada sociedad se establecen las relaciones de género, dando cuenta de un pacto social de género que indica las posiciones, los roles y las valoraciones de lo femenino y lo masculino, así como de los sujetos y cuerpos que se asocian a cada categoría. Es preciso también incluir los esfuerzos por transformar este paradigma, así como sus consecuencias.

Uno de los argumentos importantes que se debe considerar respecto del llamado privilegio epistémico de los hombres blancos occidentales es que este se funda o se sostiene no en su "excelencia" o mayor capacidad sino en la destrucción y desaparición de otras formas de producción de conocimientos y reflexiones. Se trataría de la destrucción violenta de cuatro grandes estructuras de conocimiento del sistema-mundo, cuatro genocidios del siglo XVI a los que Boaventura de Sousa Santos (2010) llama "epistemicidio":

"La epistemología occidental dominante fue construida a partir de las necesidades de la dominación capitalista colonial y se asienta en lo que designo pensamiento abismal. Este pensamiento opera por la definición unilateral de líneas radicales que dividen las experiencias, los actores y los saberes sociales entre los que son visibles, inteligibles o útiles (los que quedan de este lado de la línea) y los que son invisibles, ininteligibles, olvidados o peligrosos (los que quedan del otro lado de la línea)" (Sousa Santos, 2010: 8). 
Dichas decisiones implican la anulación y destrucción de conocimientos que quedan, como se ha señalado más arriba, del otro lado de la línea, así como la destrucción de personas, es decir, genocidios (Grosfoguel, 2013), dando origen a las formas de conocimiento modernas, asociadas al surgimiento del capitalismo (Federici, 2010). La destrucción del conocimiento, así como de las comunidades que los sustentaban, se refiere principalmente a:

a. destrucción del conocimiento de musulmanes y judíos en la conquista de

b. Al-Andalus e imposición de la episteme propia de la Iglesia Católica del siglo $\mathrm{XV}$,

c. b) contra los pueblos indígenas, primero en el continente americano y luego los aborígenes en Asia producto de la colonización,

d. c) contra los africanos, con el comercio de cautivos y su esclavización en el continente americano y

e. d) contra las mujeres que practicaban y transmitían el conocimiento indoeuropeo en Europa, quienes fueron quemadas vivas acusadas de brujas (destrucción de la corpo-política del conocimiento) en los siglos XVI y XVII.

Elgenocidio/epistemicidio perpetrado contra las mujeres indo-europeas, quienes dominaban formas de conocimiento sobre diferentes áreas como astronomía, medicina, biología, ética, entre otros ámbitos del conocimiento; también es una estrategia encarnada para terminar con el empoderamiento y los roles de liderazgo que ejercían en la organización económica, política y social. La persecución de estas comenzó a finales de la época medieval, pero se intensificó en los siglos XVI y XVII con el auge de las estructuras de poder "modernas/coloniales capitalistas/ patriarcales" (Federici, 2010). Millones de mujeres fueron quemadas vivas bajo acusaciones de brujería en el periodo moderno inicial.

De este modo, cuando en el siglo XVIII las universidades se transforman de universidades teológicas cristianas a universidades seculares kantianas/ humboldtianas (Grosfoguel, 2013), el cuerpo y la episteme de las mujeres no estaba; y de manera paralela se establecía al hombre blanco como el cuerpo y episteme de la razón, como la posición de sujeto "pensador/pensante". De este modo, se consolidaba lo que las teóricas feministas denominan como "universidad patriarcal" (Ballarín, 2015) y "razón patriarcal” (Amorós, 1985). Cuando se funda, por así decirlo, la universidad "moderna", las mujeres estaban excluidas, fuera. De ahí en más, la reivindicación ha sido por estar y por habitar la universidad durante el largo periodo en que esta universidad moderna se estructuró, definió sus áreas y temas, creó sus formar de construir conocimiento y legitimarlo. De ahí que el ingreso a las universidades implica para las mujeres y otros grupos excluidos una demanda 
que se sitúa en el horizonte de los derechos y no del conocimiento. Esta exclusión se configura de manera tan profunda en la vida social, que las demandas educativas constituyen una etapa relevante en el largo proceso político de construcción de nuevas formas de ciudadanía y participación para las mujeres (Valcárcel, 2001). Dicho de otro modo:

"La ausencia histórica de las mujeres de los centros de producción del conocimiento ha tenido una doble consecuencia: por un lado, su experiencia no forma parte del conocimiento construido, y por otro, los mecanismos sobre los que este se ha desarrollado impiden considerar su contribución sin una previa deconstrucción del primero. La contradicción entre el papel central y activo de las mujeres en el desarrollo de la sociedad y de la historia, y su marginalidad en el proceso de categorización intelectual y de creación simbólica, se ha convertido hoy en una fuerza dinámica que lleva a las mujeres a luchar desde la academia contra esta situación” (Ballarín, 2015, p.21).

Llegar tarde ha implicado que históricamente las mujeres han debido aprender las reglas y aceptarlas, generar estrategias, tanto individuales como colectivas, para superar o al menos sortear la sospecha que se instala sobre sus capacidades. Para no irnos muy lejos, en Chile, el Decreto Amunátegui² (1877) señalaba que las mujeres debían someterse a las "mismas disposiciones a que están sujetos los hombres".

En sus inicios, las universidades eran instituciones exclusivamente masculinas:

"A mediados del siglo XVII, la Universidad alemana de Utrecht autorizaba el ingreso de Anna Maria von Shuurman, aunque con una condición: durante el tiempo de aula debía permanecer encerrada "en un cuarto de madera colocado dentro de la misma aula universitaria, separado por una pared de madera en la que se habían practicado unos agujeros" (de Laurentis, 2000:12-13)" (Citado en Graña, 2008).

Este hecho recuerda lo vivido en Chile por Eloísa Díaz, la primera mujer que ingresó a la universidad a estudiar Medicina y que asistía a clases detrás de un biombo. El cuerpo de las mujeres es el mismo texto de su conocimiento (Federici, 2010) y para que este no contamine, no se expanda sobre la razón patriarcal (Amorós, 1985), debe mantenerse entonces separado, constreñido y castigado.

2. Luis Miguel Amunátegui era el ministro de Instrucción Pública del gobierno de Aníbal Pinto cuando se firmó el decreto. 
Volviendo a Chile, la promulgación del Decreto Amunátegui no cambió la situación del acceso de las mujeres a la universidad de manera inmediata y como suele suceder aún en nuestros días, los cambios en los marcos legales no necesariamente impulsan o promueven modificaciones en las prácticas culturales; desde la firma del decreto pasaron cuatro años para que una mujer entrara a la Universidad de Chile. "No obstante, las mujeres no ingresaron en masa a las aulas universitarias. Hubo años en que no ingresó ninguna (1882, 1884, 1888)" (Sánchez, 2006: 525).

La vida cotidiana de las primeras mujeres que ingresaron a la Universidad en Chile $^{3}$ se vio fuertemente entretejida con las ideologías de género que construían discursos sobre la inferioridad e incapacidad femeninas y además instalaban estrategias de naturalización de las mismas, por lo que desmontar el sexismo en la educación superior requiere hacerse cargo no sólo de las condiciones actuales sino de los procesos históricos que determinaron la exclusión de las mujeres de estas instituciones, así como de los procesos que intermediaron en las formas de legitimización de un tipo de conocimiento e instauraron la razón patriarcal. Además, en la actualidad sabemos que no son sólo las mujeres quienes enfrentan estos problemas sino todos quienes desafían el modelo de relaciones sociales heteronormativo, que se imbrica con la razón patriarcal antes mencionada y que configura parte de los códigos de género de las universidades.

\section{II.DECONSTRUCCIÓN DE LOS CÓDIGOS DE GÉNERO}

\section{II.1. Desarticulación del androcentrismo}

En esta segunda sección mostraré, a modo de ejemplo, cómo ya desde la década de 1970 emerge una mirada epistémica feminista sobre el corpus del conocimiento antropológico que muestra de qué modo este tipo de ejercicios fue y sigue siendo necesario para transformar los códigos de género que promueven desigualdades y diferentes formas de violencias en las universidades.

Como disciplina de las ciencias sociales, la antropología se ha abocado al estudio de las sociedades humanas y tal vez el concepto de cultura sea el que más la identifique. En la larga discusión desarrollada al respecto se ha intentado entre otras cosas diferenciar lo natural (biológico) de lo cultural (creado por los seres humanos), por lo que uno de sus intereses ha sido esclarecer hasta dónde ciertas

3. La Universidad de Chile fue fundada el año 1842, mientras que la Pontificia Universidad Católica, el año 1888. 
características y conductas humanas son aprendidas mediante la cultura o si están ya inscritas genéticamente en la naturaleza humana" (Lamas, 1986: 174).

Durante la década del 1960, la temática de la mujer primero, y luego la de género se articula en la antropología como producto de una discusión sobre la "ausencia" e "invisibilidad" de las mujeres en los estudios de las ciencias sociales (Lamphere, 1991; Moore, 1991). Se sostiene que la producción etnográfica presenta sesgos interpretativos que tienen que ver con la lógica cultural europea $\mathrm{y} / \mathrm{u}$ occidental (Moore, 1991; Harris y Young, 1979). La antropología, como forma de conocimiento occidental, en su articulación sistemáticamente silencia a grupos minoritarios entre los que se cuentan las mujeres, lo que forma parte de sus códigos de género, pero además constituye un sesgo epistémico, es decir, pone entre la espada y la pared las pretensiones de objetividad científica.

El silenciamiento de las mujeres se conoce como androcentrismo del conocimiento, que es necesario distinguir de situaciones como el sexismo y el machismo,

En 1975, Edwin Ardener sostuvo que la antropología forma a sus investigadores hombres y mujeres en un idioma masculino, lo que tendría como consecuencia que en el trabajo de campo se tendría mayor facilidad para captar los modelos explicativos de informantes varones, equiparando la visión masculina con la visión de la sociedad, lo que eliminaría los modelos que entregan las mujeres, pues no se dispone de herramientas conceptuales para entender este particular punto de vista (Edwin Ardener citado por Moore, 1991). La ausencia de las mujeres, en tanto grupo silenciado, también ocasionó una reacción para paliar esta ausencia, rescatarlas e incluirlas y reflexionar sobre sus roles en diferentes sociedades. A estos estudios se les ha criticado su insistencia en plantear la subordinación universal de la mujer (Montecino y Rebolledo, 1996), generando la categoría sociológica mujer, que a la par de tener ventajas traería también problemas, como anular las diferencias culturales e históricas en las posiciones y condiciones sociales de las mujeres y, de este modo, también reforzar la relación entre la posición social de las mujeres con argumentos biológicos.

Rescatar la categoría de mujer en estas discusiones fue el primer paso desde donde se determinaron una serie de problemas teóricos, metodológicos y prácticos que hicieron necesario avanzar en la discusión de un modo más profundo. Es por esto que parece más adecuado usar el concepto de género, entendido básicamente como la construcción social de las diferencias biológicas entre hombres y mujeres en cada sociedad, realzando la importancia que poseen las "configuraciones culturales" en la determinación de los roles, normas, actitudes, etc., que uno y otro género desarrolla en contextos culturales específicos. La discusión no sólo se centra en la diversidad cultural del fenómeno sino en la preponderancia de la cultura en la determinación del mismo: esto determinó el uso del concepto de género por parte 
de la antropología por varias décadas para dar cuenta de la construcción social y cultural de las diferencias sexuales que establece una sociedad. Se trata por ello, de una realidad dinámica, que varía según la clase, la edad, el momento histórico y por sobre todo, de sociedad en sociedad, pero con una perspectiva feminista a fin de no caer en el juego de relativismo cultural sino que introduciendo una perspectiva crítica sobre los derechos de las mujeres y las transformaciones culturales que se imponen en este ámbito, sobre todo a partir de la consolidación de la globalización cultural (Segato, 2015).

La utilización analítica del concepto de género debería implicar necesariamente dejar de concebir la construcción cultural de los géneros de un modo binario, es decir, como si existieran un femenino y un masculino de modo absoluto en las sociedades. Las mismas contextualizaciones de su utilización determinan que en una sociedad puedan coexistir múltiples y paralelas construcciones sociales de lo femenino y lo masculino. El uso del concepto de género dio a la antropología la posibilidad de entender la posición diferencial de hombres y mujeres en una misma sociedad y en sociedades diferentes, considerando tanto los factores culturales o simbólicos y los socioeconómicos.

\section{II.2. Valorización del conocimiento feminista y el debate de los estudios de género}

La exclusión y la razón patriarcal han puesto entre comillas la participación de las mujeres en los procesos de construcción de conocimiento científico y, por lo tanto, también en las universidades. Como se ha dicho más arriba, su presencia requiere de formas de legitimación. La reproducción de las inequidades de género en el sistema educativo es un problema que fue situado hace ya varias décadas por investigadoras feministas, quienes además establecieron hipótesis. Resulta evidente que el señalamiento del sexismo en la educación forma parte de la teoría feminista para discutir sobre este problema y requiere hacerse dentro de este marco. Además, existen diferentes aproximaciones y definiciones que alumbran diferentes niveles del problema (Thompson, 2003).

No obstante la importancia del conocimiento feminista y la circulación y apropiación de sus conceptos, existen reacciones y rechazos al conocimiento crítico del feminismo que se expresan de varias maneras. Primero, no considerarlo científico por el compromiso político de las pensadoras y pensadores feministas, dado que entonces no sería "objetivo"; se establece entonces una suerte de tensión y/o disputa que sitúa a los conocimientos surgidos en el feminismo como productos ideológicos, de carácter social, pero no científicos. Segundo, existe reticencia a 
incorporar en los planes de estudio materias específicas relacionadas con género, mujeres, o feminismo. Además, se tiende a la marginalidad en el proceso de categorización intelectual y de creación simbólica, que se ha convertido hoy en una fuerza dinámica que lleva a las mujeres a luchar desde la academia contra esta situación. La docencia, en la mayoría de las disciplinas universitarias, mantiene los supuestos teóricos y metodológicos androcéntricos, ignorando los conocimientos aportados desde el feminismo y los análisis de género (Ballarín, 2015).

\section{II.3. Las condiciones del habitar}

Finalmente, otra de las aristas del sexismo en la educación se constituye también a través y junto con lo que denomino las condiciones del habitar, es decir, las condiciones estructurales, materiales y simbólicas en las que se desenvuelven las mujeres y grupos en disputa con el modelo heteronormativo en las universidades, dentro de las cuales cuento la valoración material del trabajo (salario); la construcción de las dinámicas de prestigio, su acceso y distribución entre los miembros de la comunidad, estos dos factores indicen en las formas de valoración y representación de las mujeres y otras comunidades que denominaríamos "grupos silenciados en las universidades; los mecanismos de la carrera académica desde la etapa estudiantil; la violencia de género: acoso sexual laboral, acoso y abuso sexual, cuyas víctimas más vulnerables son las estudiantes; y ausencia de un debate sobre la diversidad sexual.

Algunos de los temas anteriores han sido al menos develados en el conjunto del sistema de educación superior latinoamericano y chileno, tales como las desigualdades salariales y las dinámicas de género en las trayectorias laborales académicas. Un ejemplo es el estudio Del biombo a la cátedra (Universidad de Chile, 2013), que aborda los aspectos centrales para avanzar en el momento actual. Estos se relacionan con la reproducción de la violencia material de género en todas las estructuras universitarias, es decir, aquella que afecta en el trato diario a las personas, sean estas funcionarios(as) académicas(os) y/o estudiantes, que en los últimos años ha abierto el debate respecto del acoso sexual y laboral en recintos universitarios y que constituye una de las expresiones más claras de la constitución de las universidades como instituciones generizadas. Este abordaje también permite enfrentar la pregunta sobre cómo desmontar esta violencia, entendida también como un dispositivo de control y discriminación, y cómo podemos darle un lugar en el sistema de enseñanza-aprendizaje con el objetivo de desmontar estos comportamientos.

Como he señalado anteriormente, las teóricas feministas han observado cómo el sistema educativo legitima las diferencias de género a través de lo explícito del sistema y por su imbricación en la reproducción de las desigualdades de género 
propias de la sociedad y la cultura. Como hemos señalado más arriba, parte de lo explícito del sistema refiere tanto a los enfoques teóricos utilizados como a la falta de perspectivas críticas o la inclusión de las mismas, el modelo formativo, las bases curriculares y la malla curricular. En las universidades, del mismo modo que en las escuelas básicas y secundarias, se articula un currículo oculto de género que no enseña sino que disciplina respecto de cuáles son los conocimientos que están del lado apropiado de la línea -parafraseando a Boaventura de Souza Santos-, indicando el lugar para las mujeres, insistiendo en la valoración del modelo heteronormativo y permitiendo y delineando el modo de ingreso de conocimientos, sujetos e identidades en disputa con dicho modelo. Tal vez es posible seguir enumerando prácticas y condiciones que articulan el currículo oculto, pero lo que queremos puntualizar, más bien, es que se trata de relaciones de micro poder que se despliegan por todo el sistema, en todos sus niveles y a través de todos sus actores. Dicho esto, es necesario recalcar que sus efectos son contundentes y perjudiciales para el sistema.

\section{CIERRE}

El recorrido propuesto en este artículo esboza algunos de los elementos centrales para discutir sobre cómo podemos seguir avanzando en la equidad de género en las universidades chilenas. En primer lugar, es necesario pensar que la exclusión de las mujeres es más que una exclusión física, material e histórica: es la expresión de un problema epistémico relacionado con la valoración y aceptación de ciertas formas de pensamiento y la eliminación (epistemicidio) de otras que determinan una homologación entre estos conocimientos y los sujetos que los construyen y reproducen.

Este proceso funda el orden de género de las universidades interdigitadamente con el conocimiento científico occidental. Durante las décadas de 1960 y 1970, el movimiento por los derechos de las mujeres también tuvo una expresión académica, universitaria, que tuvo como uno de sus objetivos centrales la deconstrucción del androcentrismo teórico, así como reforzar y finalmente crear centros de estudios orientados a construir conocimiento sobre las mujeres en una primera instancia, sobre las relaciones de género en un segundo momento, y sobre las diversidades sexuales en la actualidad. Sin embargo, aunque estos procesos transformaron las bases del orden de género de las universidades, no lograron desmontar ni el sexismo ni el androcentrismo del conocimiento en las estructuras universitarias, pero se constituyen en una base importante para avanzar. Uno de los aspectos más importantes, que sigue sin desmontarse, es la violencia cotidiana, no sólo expresada 
en prejuicios sobre las capacidades cognitivas sino como abusos y acoso sexual. Lo que no sólo es una vulneración a los derechos de las mujeres sino que es una forma de cercar nuevamente a las mujeres.

Un primer esfuerzo debería ser pensar en un diagnóstico sobre el orden de género en las universidades, y es asumir que cada institución universitaria expresa dicho orden de manera particular, con códigos y prácticas específicas, pero es necesario conocerlo, observarlo y deconstruirlo. En segundo lugar, es necesario recoger las herramientas que el propio pensamiento feminista ha puesto en marcha décadas atrás para pensar y revisar la historia de muchas disciplinas, del conocimiento científico y sobre la ciencia. Es necesario pensar en mujeres que fueron pioneras en muchas áreas y cómo sus llegadas también cambiaron las formas legítimas del pensamiento. Estos dos niveles deberían ser más centrales en la transformación de la educación y por qué no, la erradicación de los estereotipos de género. Sin embargo, de todas maneras esto resulta insuficiente, pues se requiere que el conjunto de la comunidad académica pueda entender de qué se trata y a qué apuntan y cuáles son sus impactos tanto en los resultados de los aprendizajes como en la investigación, así como en las personas de manera concreta.

Finalmente, es necesario abordar el hecho de que en la actualidad se requieren cada vez más conocimientos e informaciones sobre la vida de las mujeres, las rearticulaciones de las violencias contra ellas, las maneras en que se expresan las desigualdades de género, la amenaza a los derechos de las mujeres. Una educación no sexista no debe obviar este horizonte teórico-ético que implica tanto investigar como formar para transformar la vida de las mujeres y con las mujeres. 


\section{REFERENCIAS}

CORDERO, T. Mujeres transformando mandatos sociales (1940-1959). San José, UCR, 2014.

CUBILLOS, S.; Monreal. A. Psiquiatras chilenas: Pioneras en la locura. Santiago, Ediciones Radio Universidad de Chile, 2013.

Ballarín, Pilar. Los códigos de género en la universidad. En: Revista Ibero-americana de Educação, vol. 68:19-38, 2015.

FEDERICI, Silvia. Calibán y la bruja. Mujeres, cuerpo y acumulación originaria. Madrid, Traficantes de Sueños, 2010.

FLECHA, C. (2010). Profesoras en la Universidad. El tránsito de las pioneras en España. En: Revista Arenal, Vol. 17, N²: 255-297.

GRAÑA, Francois. El asalto de las mujeres a las carreras universitarias "masculinas": cambio y continuidad en la discriminación de género. En: Praxis, N¹2: 7786, 2008.

GROSFOGUEL, Ramón. Racismo/sexismo epistémico, universidades occidentalizadas y los cuatro genocidios/ epistemicidios del largo siglo XVI. En: Tabula Rasa. Bogotá - Colombia, No.19: 31-58, 2013

LAMAS, Marta. La antropología feminista y la categoría de género. En: Nueva Antropología, Vol. VIII, No 3: 173-198, 1986.

LAMPHERE, Louise. Feminismo y antropología. En: El género en perspectiva, de la dominación universal a la representación múltiple. Universidad Autónoma Metropolitana, México D.F., 1991: 279315.

HARRIS, O. y Kate Young. Introducción de Antropología y Feminismo, Ed. Anagrama, Barcelona, 1979: 932.

MAGALLÓN, C. Las mujeres que abrieron los espacios de las ciencias experimentales para las mujeres en la España del primer tercio del siglo XX. En: Revista Arenal, Vol. 17, N²: 319-347, 2010.

MINGO. A. ¿Quién mordió la manzana? Sexo, origen social y desempeño en la Universidad. PUEG, UNAM. Ciudad de México: Fondo de Cultura Económica, 2006.

MONTECINO, S. y Rebolledo, L. Conceptos de género y desarrollo. Santiago, PIEG, Universidad de Chile, 1996.

MOORE, H. Antropología y Feminismo. Ed. Cátedra, Universitat de Valencia, Instituto de la Mujer, Madrid, 1991. 
UNIVERSIDAD DE CHILE. Oficina de Igualdad de Oportunidades de Género. Del biombo a la Cátedra. Igualdad de Oportunidades de Género en la Universidad de Chile. Santiago, Rectoría, Universidad de Chile, 2014.

SEGATO, Rita. La crítica de la colonialidad en ocho ensayos. Una antropología por demanda. Buenos Aires, Prometeo Libros, 2015.

SÁNCHEZ, K. El ingreso de la mujer chilena a la Universidad y los cambios en la costumbre por medio de la ley 1872-1877. Historia (Santiago), 39(2): 497-529, 2006. Recuperado en 05 de noviembre de 2014, de http://www.scielo.cl/ scielo.php?script $=$ sci_arttext\&pid $=$ S0717-71942006000200005\&lng $=$ es\&tln $\mathrm{g}=$ es. $10.4067 / \mathrm{S} 0717-71942006000200005$.

SOUSA SANTOS, B. Descolonizar el saber, reinventar el poder. Montevideo, Ediciones Trilce, 2010.

WOLFF, X.; Winkler, M.I.; Alvear, K. Subversiones de género: pioneras de la psicología chilena y estadounidense. En: Psychologia Latina, 2010, Vol. 1 No. 2: 129-138, 2010. 